\title{
Airway dimensions measured from micro- computed tomography and high-resolution computed tomography
}

\author{
J.R. Dame Carroll*,\#, , A. Chandra*,\#, , A.S. Jones ${ }^{+}$, N. Berend*,\#, , \\ J.S. Magnussen*,§ and G.G. King*,\#,ף,f
}

ABSTRACT: Volume averaging results in both over- and underestimation of airway dimensions when they are measured by high-resolution computed tomography (HRCT). The current authors calibrated computerised measurements of airway dimensions from HRCT against a novel threedimensional micro-computed tomography (CT) standard, which has a 50 -fold greater resolution, as well as against traditional morphometry.

Inflation-fixed porcine lung cubes were scanned by HRCT and micro-CT. A total of 59 lumen area $\left(A_{i}\right), 30$ wall area $(A a w)$ and 11 lumen volume $\left(V_{i}\right)$ measurements were made. Ai was measured from the cut surface of 11 airways by morphometry. Airways in scanned images were matched using branching points. After calibration, the errors of $A i$, Aaw and Vi HRCT measurements were determined.

The current authors found a systematic, size-dependent underestimation of $\mathrm{Ai}$ and overestimation of Aaw from HRCT measurements. This was used to calibrate an HRCT measurement algorithm. The $95 \%$ limits of agreement of subsequent measurements were $\pm 3.2 \mathrm{~mm}^{2}$ for $\mathrm{Ai}$, $\pm 4.3 \mathrm{~mm}^{2}$ for Aaw, and $\pm \mathbf{1 1 . 2} \mathrm{mm}^{3}$ for $\mathrm{Vi}$ with no systematic error. Morphometric measurements agreed with micro-CT $\left( \pm 2.5 \mathrm{~mm}^{2}\right)$ without systematic error.

In conclusion, micro-computed tomography image data from inflation-fixed airways can be used as calibration standards for three-dimensional lumen volume measurements from high-resolution computed tomography, while morphometry is acceptable for two-dimensional measurements. The image dataset could be used to validate other developmental three-dimensional segmentation algorithms.

\section{KEYWORDS: Computer-assisted image processing, imaging, phantoms, validation studies}

D ue to multidetector technology, volumetric high-resolution computed tomography (HRCT) image data are routinely acquired, and have been used for quantitative image analysis of airways and lung tissue in many studies [1-9]. Direct measurements of airway structures can be made from HRCT image data, making it useful for studying asthma, cystic fibrosis and chronic obstructive pulmonary disease. Airway dimensions have been measured from HRCT image data using customised computer-analysis algorithms that have been validated using airway phantoms of known dimensions constructed from nonanimal materials, such as plexiglass and sweet potato $[4,8,10-$ 12]. In these studies, planimetric measurements were made of a cut surface of the phantom. It was therefore reasonably assumed that the dimensions would be completely uniform along the length of the airway phantom and that orientation in the z-axis of the scanning plane could be accurately controlled.

Validation of airway measurement algorithms advanced further with the use of explanted animal lungs as calibration standards. Porcine lungs wet fixed with formalin [13] have been used and the current authors have previously used formalin inflation-fixed porcine lungs [8] for validation. The use of liquid-fixed resected human lung tissue for validation has also recently been described [13]. Explanted lung preparations are more suitable than artificial phantoms for validation because they closely approximate the range of tissue densities and dimensions, and the complex structure of lung tissues in vivo. This includes features such as mucosal folding and irregularities in airways, which are not present in artificial phantoms. This
AFFILIATIONS

*The Woolcock Institute of Medical Research, and

\#The Cooperative Research Centre for Asthma, and

"University of Sydney, and

+The Key Centre for Microscopy and Microanalysis, University of Sydney, Sydney, and

${ }^{\text {s}}$ Dept of Radiology, Royal Prince Alfred Hospital, Camperdown, and ${ }^{f}$ Dept of Respiratory Medicine, Royal North Shore Hospital, St. Leonards, Australia.

CORRESPONDENCE

G.G. King

The Woolcock Institute of Medical Research

University of Sydney

Sydney 2006

Australia

Fax: 61299066391

E-mail: ggk@woolcock.org.au

Received:

February 032005

Accepted after revision:

June 302006

\section{SUPPORT STATEMENT}

This study was supported by the Cooperative Research Centre for Asthma (Project 12b) and National Health and Medical Research Council Project Grant No. 153839.

European Respiratory Journal Print ISSN 0903-1936 Online ISSN 1399-3003 
a)

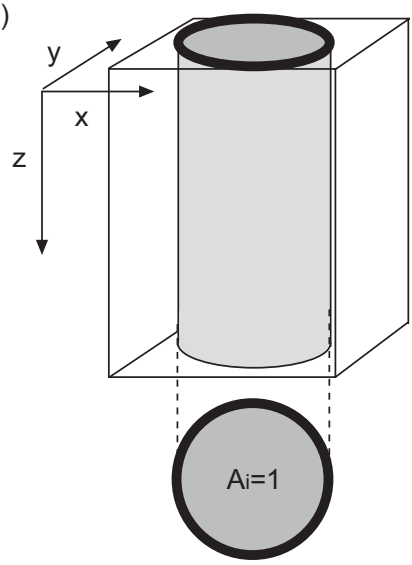

b)

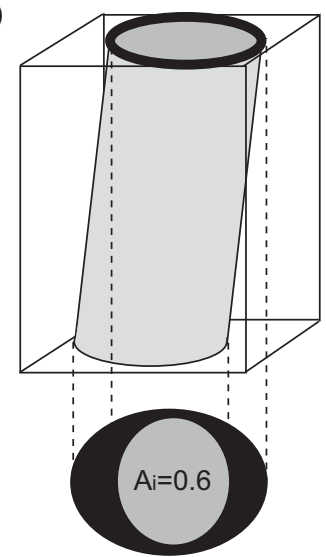

FIGURE 1. Theoretical effect of volume averaging on internal lumen area (Ai) measurements made from high-resolution computed tomography images. a) When airways are parallel to the $z$-axis of the scanner, no volume averaging occurs and the cross-sectional area is theoretically accurately measured; b) when an airway is oriented at an angle in relation to the $z$-axis, volume averaging of the airway wall occurs resulting in an apparently smaller $\mathrm{Ai}$.

difference compared with artificial material phantoms is, in turn, associated with different estimates of measurement errors for HRCT [8]. HRCT measurements are affected by volume averaging, particularly in the scanner's z-axis. This is due to the complexity of the structures, range of tissue densities, inability to control airway orientation in the z-axis during scanning, and the fact that the $\mathrm{z}$-axis is the longest dimension of the voxel (fig. 1). To calibrate three-dimensional (3D) HRCT measurements of airways, the current authors required a $3 \mathrm{D}$ standard of known dimensions so that the effects of volume averaging would be accounted for in the calibration process, while also allowing measurement of the precision and accuracy of the HRCT method. As morphometry is twodimensional (2D), there is by definition no volume averaging in the $\mathrm{z}$-axis to affect this measurement.

Micro-computed tomography (CT) is currently used for studying the $3 \mathrm{D}$ structure of a wide range of small objects in microscopic detail with image data consisting of cubic voxels as small as $2 \times 2 \times 2 \mu \mathrm{m}$, but its use in the lung has not been widely explored. Micro-CT resolution is almost two orders of magnitude greater than HRCT, in which voxel size is typically $0.5 \times 0.5 \times 1 \mathrm{~mm}$. The resolution of micro-CT should allow airway measurements of similar or greater accuracy to morphometry, but in three dimensions, which would allow validation of $3 \mathrm{D}$ segmentation algorithms used to measure airway dimensions in vivo (fig. 2). The primary aim of the present study was to validate micro-CT against the current calibration standard of morphometry of the cut surface of the lung and calliper measurement of cylindrical rods. The secondary aim was to use measurements of lumen area $(\mathrm{Ai})$ and wall area (Aaw) in inflation-fixed porcine airways from micro-CT images to calibrate an HRCT airway segmentation algorithm. The final aim was to assess the precision and accuracy of 3D measurements of airway volume ( $\mathrm{Vi}$ ) made using the calibrated segmentation algorithm.
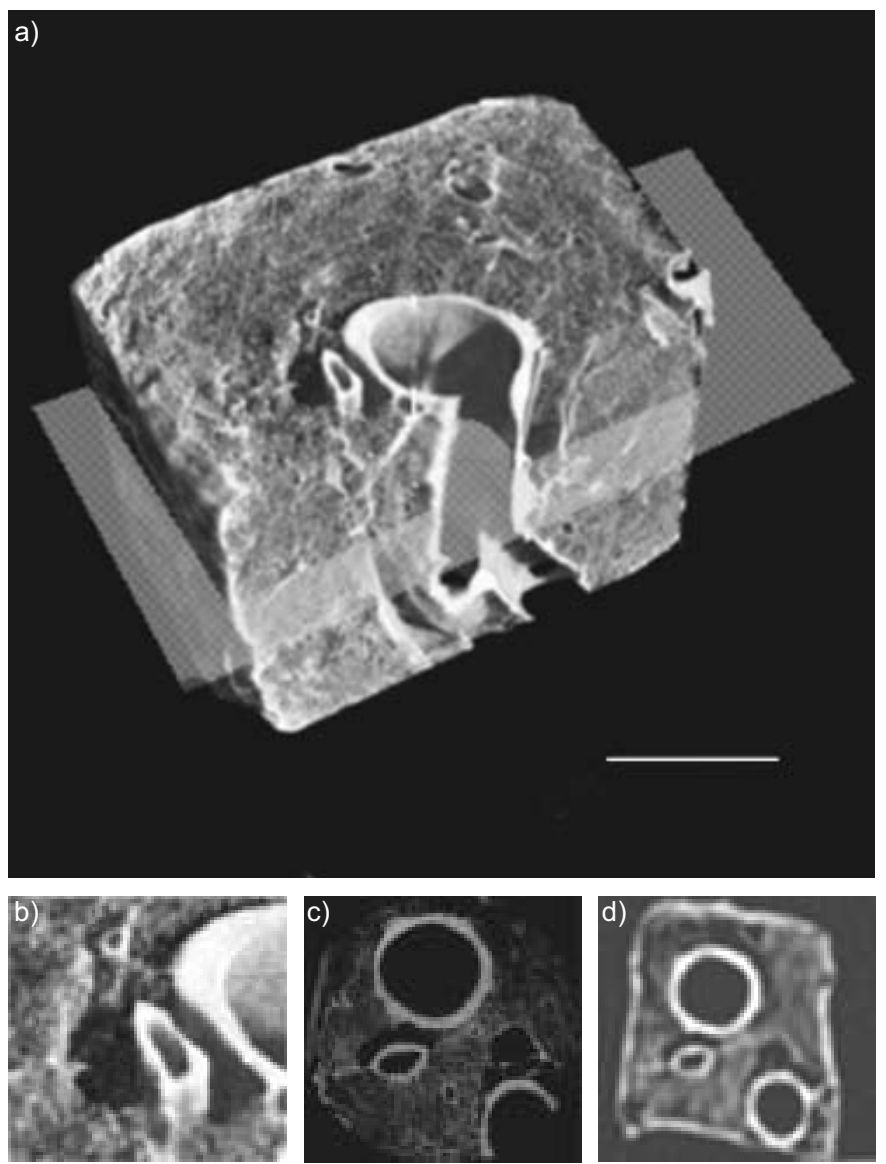

FIGURE 2. a, b) Surface-rendered three-dimensional micro-computed tomography (CT) image of a pig-lung cube; c) cross-sectional image; and d) highresolution $\mathrm{CT}$ image of the same cube. Scale bar $=1 \mathrm{~cm}$.

\section{METHODS}

To validate micro-CT as the calibration standard for an HRCT airway segmentation algorithm, micro-CT diameter measurements of plastic and wooden cylindrical rods of various sizes were compared with calliper measurements. Ai measurements from a single micro-CT image of the cut surface of inflationfixed porcine lung were compared with the current gold standard, tissue morphometry [8, 14]. Micro-CT measurements of $\mathrm{Ai}$ and Aaw were used to calibrate a segmentation algorithm for unbiased $\mathrm{Ai}$ and Aaw measurements from HRCT and the precision (95\% limits of agreement) of $\mathrm{Ai}, \mathrm{Aaw}$ and $\mathrm{Vi}_{\mathrm{i}}$ measurements of the calibrated algorithm. Finally, the degree of agreement between calibrated HRCT measurements of $\mathrm{A}$ from the cut surface of the lung and morphometric measurements was examined (fig. 3).

\section{Lung preparation and fixation}

Lungs were fixed in inflation with formalin [8] using modification of a method described by WEIBEL and VIDONE [15]. The lungs were obtained from a local butcher and were fixed $12-24 \mathrm{~h}$ after being removed from the animal. Formalin steam was passed into the lung and negative pressure was applied to the exterior of the lung to fix the lung in inflation at an approximate inflation pressure of $25 \mathrm{cmH}_{2} \mathrm{O}$ (fig. 4). 

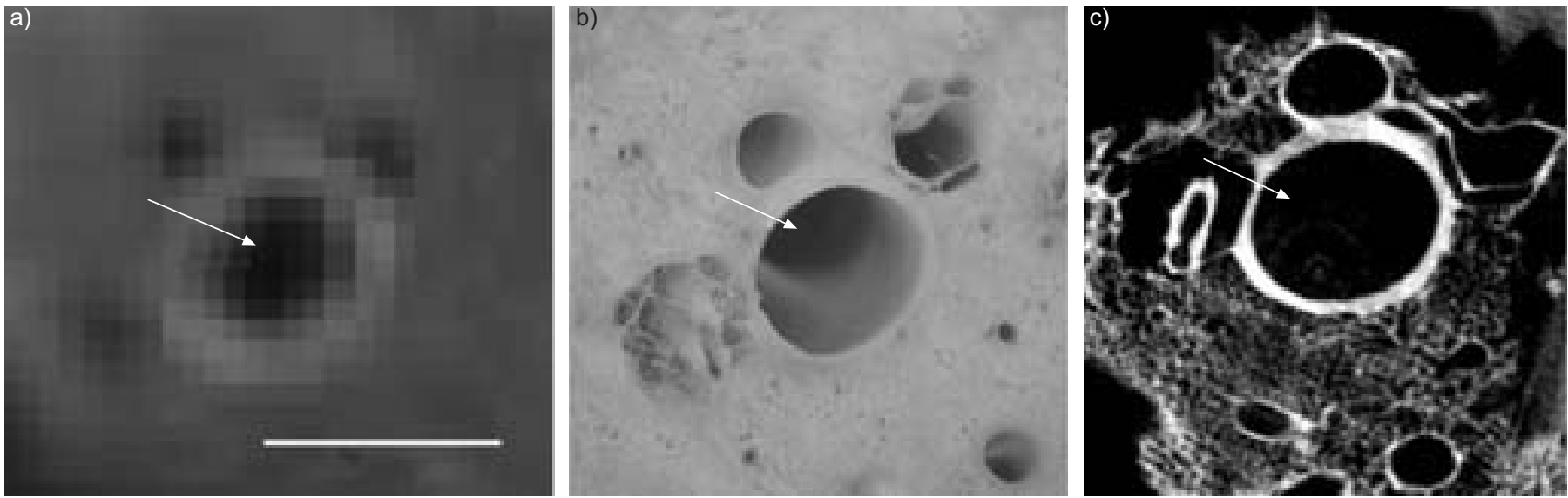

FIGURE 3. A comparison of cut surface images produced by a) high-resolution computed tomography (CT); b) digital photography; and c) micro-CT. The arrow in all three panels indicates the same airway. Scale bars $=5 \mathrm{~mm}$.

Fixation time was $\sim 2 \mathrm{~h}$, depending on the size of the specimen.

After fixation and cooling to room temperature, the lung was scanned by HRCT and airways of interest were identified. The lung was then cut into $2-\mathrm{cm}$ cubes in a plane approximately perpendicular to the $\mathrm{z}$-axis of the airways of interest. The cut surfaces of the lung cubes were photographed. Finally, the 2$\mathrm{cm}$ lung cubes were wrapped in plastic wrap and coated in paraffin for protection (Paraplast; Tyco Healthcare, Mansfield, MA, USA).

\section{Digital imaging of the lung}

Immediately prior to being wrapped and coated with paraffin, the cut surfaces of the lung cubes were photographed through a dissecting microscope with a 3.34-megapixel digital camera (Nikon Coolpix 995 with MDC2 Relay Lens; Nikon, Tokyo, Japan) and images were saved as $1024 \times 1024$-pixel bitmap files. After the lung cubes were wrapped and coated in paraffin they were scanned in both the HRCT (GE 4-slice Lightspeed; GE Medical Systems, Minneapolis, MN, USA) and SkyScan 1072 micro-CT desktop systems (SkyScan, Aartselaar, Belgium). HRCT settings were $100 \mathrm{kV}$ and $100 \mathrm{mAs}$ at a slice thickness of $1.25 \mathrm{~mm}$ and a pitch of one in helical mode. Images were reconstructed using the GE high spatial frequency "Bone" algorithm at a field of view (FOV) of $18 \mathrm{~cm}$, yielding a voxel size of $0.35 \times 0.35 \times 1.25 \mathrm{~mm}$. Images were archived in DICOM format for analyses later. Micro-CT settings were $100 \mathrm{kV}$ and $100 \mathrm{mAs}$ at a slice thickness of $19 \mu \mathrm{m}$

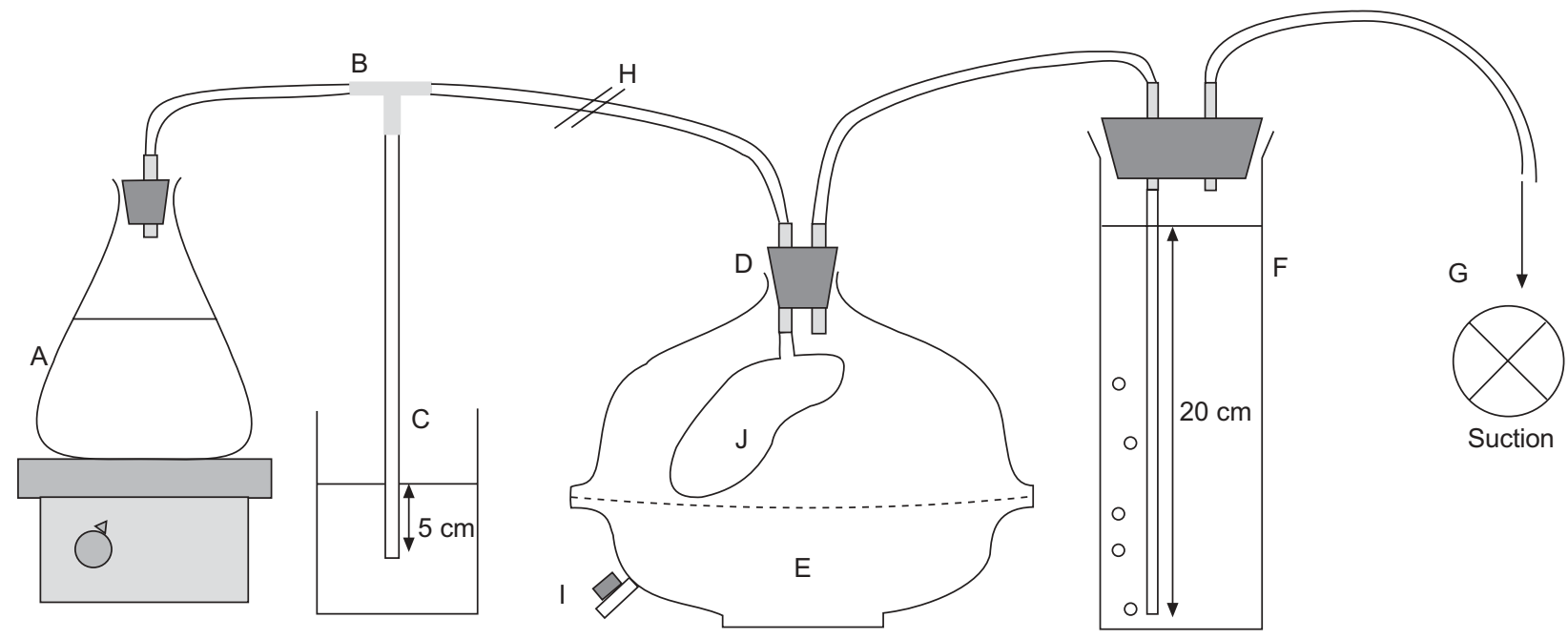

FIGURE 4. Apparatus used in formaldehyde steam fixation of a lung in inflation. The circuit was set up in a fume cupboard. The lung (j) was suspended in a specially modified Nalgene bell jar (d; Nalge Nunc, Rochester, NY, USA). Lungs were generally intact, but any visible cuts, tears or leaks on the lungs were sealed using spring-loaded paper clips. A $30 \%$ formaldehyde solution ( $a$; three parts $40 \%$ formaldehyde mixed with one part distilled water) was boiled until there was gentle bubbling from the side-arm of a t-tube (b) the open end of which was submerged in water to a depth of $5 \mathrm{~cm}$ (c). The lung was inflated by both the 5 - $\mathrm{cmH}_{2} \mathrm{O}$ positive pressure generated by the formalin steam and the $20-\mathrm{cmH}_{2} \mathrm{O}$ negative pressure provided by wall suction $(\mathrm{g})$ surrounding the lung in the bell jar, producing an overall transpulmonary pressure of $\sim 25 \mathrm{~cm} \mathrm{H}_{2} \mathrm{O}$ There was a small amount of airflow out of the lungs through small unsealable leaks in the pleural surface, with the lung acting as a resistor in the flow circuit. There was also an adjustable external "leak" (i) into the bell jar, which was used for adjusting pressures within the jar (e). Due to the variability in leaks between lung specimens, the lung "leak" was adjusted (g) so that there was gentle bubbling from the side-arm of the t-tube submerged in water to a depth of $20 \mathrm{~cm}$ (f). h: adjustable clamp. 
and a FOV of $19 \mathrm{~mm}$, yielding a voxel size of $19 \times 19 \times 19 \mu \mathrm{m}$. Each cube was scanned every $0.23^{\circ}$ as it rotated around its vertical axis until it had rotated $180^{\circ}$, at an exposure time of $0.9 \mathrm{~s}$ per scan. Images were reconstructed using the ConeRec software (SkyScan). Reconstructed image data were archived in bitmap format for analyses later.

\section{Image analysis}

A single operator (J.R. Dame Carroll) measured Ai and Aaw of airways visible in cross-section in the digital photographic bitmap images of the cut surface of the lung, manually tracing the inner and outer edges of the airway wall with the computer mouse using ImageJ software (National Institute of Mental Health, Bethesda, MD, USA). Each airway was traced twice to assess for measurement repeatability, and the average of the two tracings was used for analysis.

Ai and Aaw measurements were made directly from the HRCT image data (DICOM format) using an in-house, custom-written software program. The HRCT lung images were displayed onscreen and the airway of interest was identified manually. The centroid of the airway was automatically identified, and 20 radial spikes were created running out from it. The inner and outer edges of the airway wall were defined as the point along the radial spikes at which the greatest rate of change in density occurred, i.e. the transition from air to soft tissue.

$\mathrm{Ai}$ and Aaw were measured from micro-CT bitmap images using the same method as for morphometry, tracing the inner and outer edges of the airway wall using the ImageJ software. The lumen and wall areas were calculated as the product of the pixel size $\left(19 \times 19 \mu^{2}\right)$ and the number of voxels within the traced border. A single operator (J.R. Dame Carroll) traced each airway border twice, and the average of the two measurements was used for analysis. For comparisons of micro-CT and morphometry, all airways that were visible in both the micro-CT FOV and the digital photographs of the cut surface of the lung specimens were measured. This resulted in a range of airway sizes comparable to those measured by the current authors in a previous HRCT study of asthmatic and normal airways [16].

For comparisons between HRCT and micro-CT, airways were matched using airway branch points as anatomical markers. Since the HRCT slice thickness of $1.25 \mathrm{~mm}$ was $>60$ times thicker than the micro-CT slice thickness of $19 \mu \mathrm{m}$, every 30th micro-CT image was used to give an effective slice thickness of $570 \mu \mathrm{m}$. To calibrate Ai measurements from HRCT, the average of three consecutive $\mathrm{Ai}$ and Aaw measurements from micro-CT images which covered a thickness of $1.14 \mathrm{~mm}$, was compared with each corresponding HRCT slice of thickness $1.25 \mathrm{~mm}$. Micro-CT and HRCT measurements of Vi were calculated as the sum of all lumen voxels in the airway. Voxel dimensions for micro-CT were $19 \times 19 \times 570 \mu \mathrm{m}$, since only every 30 th image was used (30 slices $\times 19 \mu \mathrm{m}=570 \mu \mathrm{m})$. The voxel dimensions for HRCT were $0.35 \times 0.35 \times 1.25 \mathrm{~mm}$, since slice thickness was $1.25 \mathrm{~mm}$.

\section{Statistical analysis}

All data are shown as mean $\pm 95 \%$ confidence interval, unless otherwise specified.

\section{Intra-observer variation}

Intra-observer variations in 72 calliper measurements of the rods, in $\mathrm{Ai}$ and $\sqrt{\mathrm{Ai}}$ measurements from morphometry in 29 airways, and in $\mathrm{Ai}$ measurements from micro-CT in 30 airways, were determined by the methods of BLAND and ALTMAN $[17,18]$ and expressed as repeatability, being the $95 \%$ limits of agreement.

\section{Agreement between imaging methods}

The degree of agreement of the $\mathrm{Ai}$ and Aaw measurements made by the semi-automated HRCT algorithm and the microCT technique was calculated by the method of BLAND and ALTMAN $[17,18]$ and expressed as the $95 \%$ limits of agreement, being $\mathrm{t} 0.05 \times \mathrm{SD}$ of the differences between micro-CT and HRCT measurements, where $t 0.05$ is the critical $t$-value corresponding to the sample size at the 0.05 level of significance. Agreement
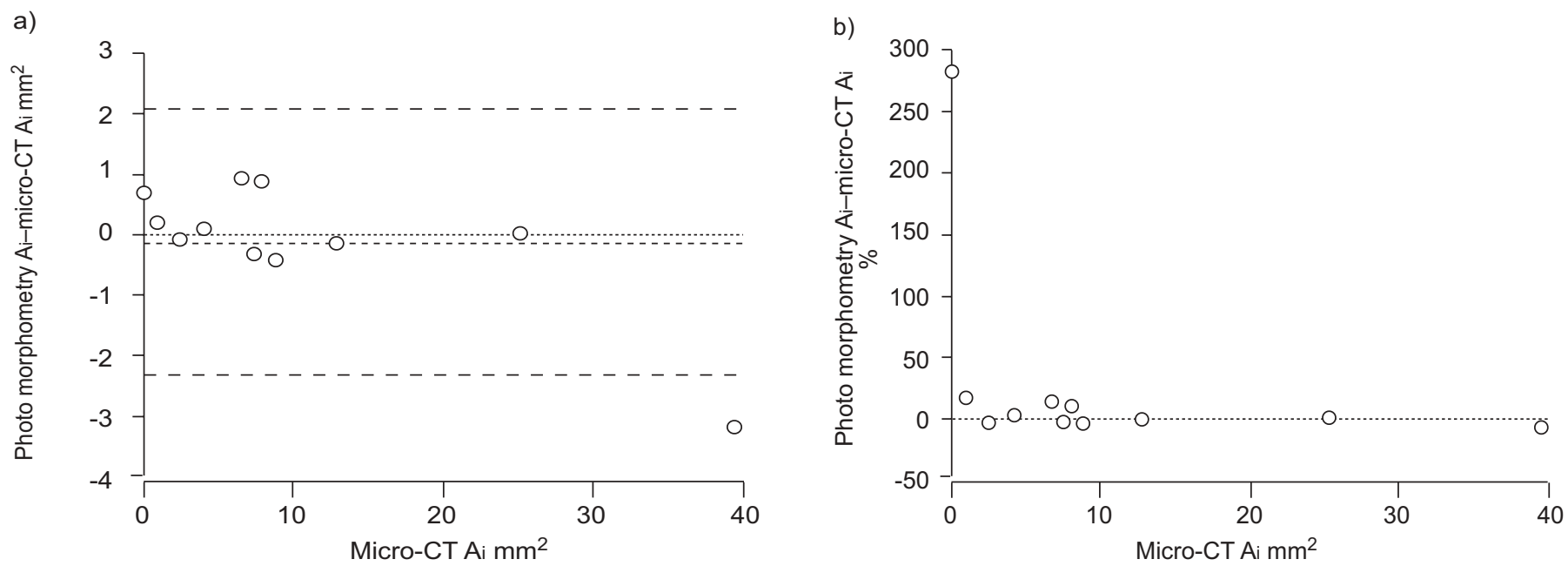

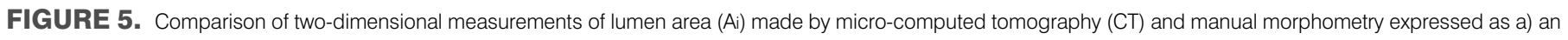
absolute difference and b) a percentage difference. ----: mean difference; - - -: 95\% limits of agreement. 
between micro-CT measurements and calliper measurements of the artificial material rods was similarly calculated.

\section{Calibration of HRCT measurements}

The relationship between $\mathrm{Ai}$ and Aaw measurements from micro-CT and HRCT was used as a calibration factor for the HRCT measurements and the subsequent 95\% limits of agreement were recalculated as above.

\section{RESULTS}

\section{Calliper measurements}

The measurements made by micro-CT were validated against 72 calliper measurements of cylindrical rods, made of artificial materials, with diameters between $0.8 \mathrm{~mm}$ and $9.8 \mathrm{~mm}$. The intra-operator repeatability of the calliper measurements of the rods was $\pm 0.21 \mathrm{~mm}$ and was independent of diameter. Consequently, the percentage error was greater for smaller rods. The mean difference between micro-CT and calliper measurements of diameter was $0.03 \pm 0.03 \mathrm{~mm}(\mathrm{p}=0.14)$ and the $95 \%$ limits of agreement were $\pm 0.285 \mathrm{~mm}$. Thus, there was no systematic error in micro-CT measurements.
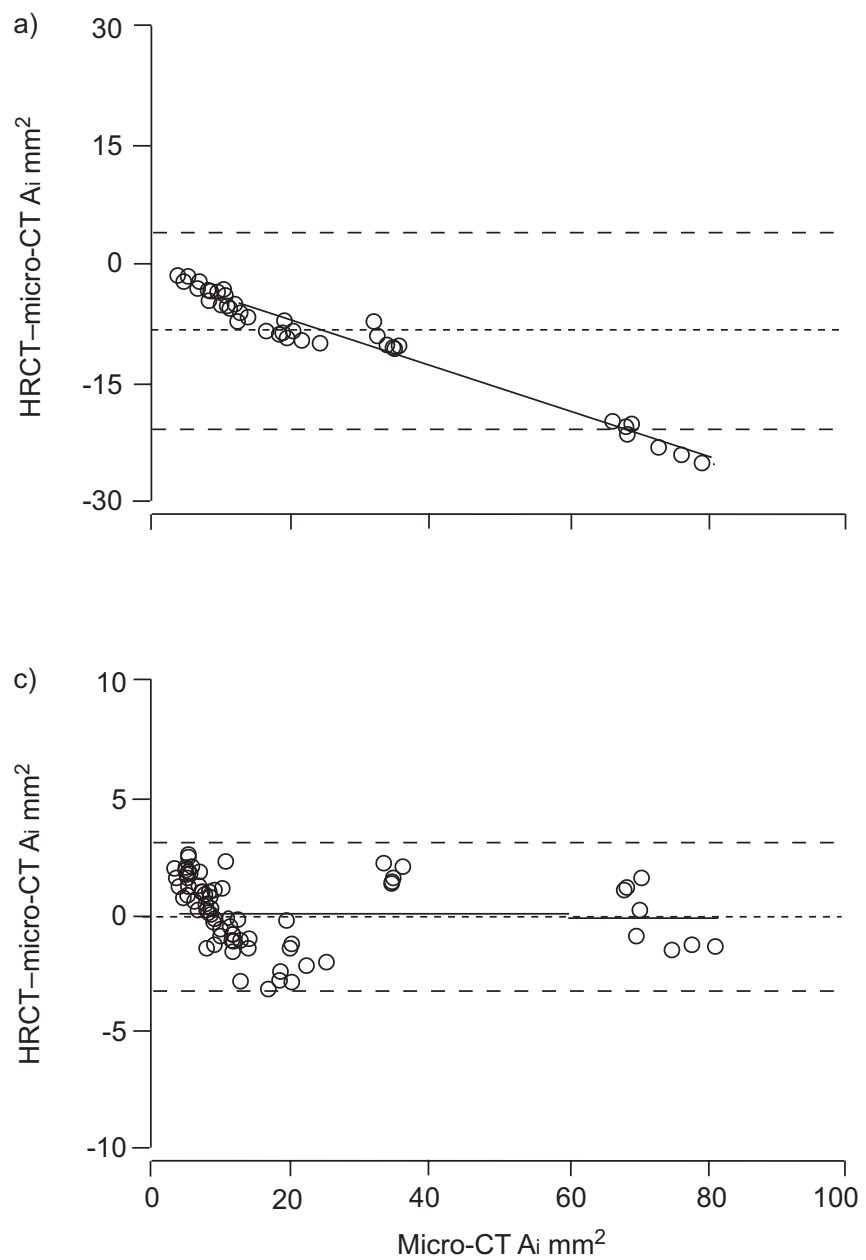

\section{Morphometry versus micro-CT of airways}

There was good agreement between Ai measurements of 11 airways from the digital photographs of the cut lung surface compared with measurements from micro-CT (fig. 5). Within the range of airway sizes the current authors were able to study $\left(0.9-36.4 \mathrm{~mm}^{2}\right)$, the mean difference was $0.14 \pm 0.6 \mathrm{~mm}^{2}$ $(p=0.69)$, independent of airway size; the $95 \%$ limits of agreement were $\pm 2.19 \mathrm{~mm}^{2}$. The intra-operator (J.R. Dame Carroll) repeatabilities of $\mathrm{Ai}$ and $\sqrt{\mathrm{Ai}}$ measurements from morphometry were $\pm 0.555 \mathrm{~mm}^{2}$ or $3.1 \%$ and $\pm 0.066 \mathrm{~mm}$ or $1.6 \%$, respectively. The intra-operator repeatability of $\mathrm{Ai}$ measurements from micro-CT was $\pm 0.557 \mathrm{~mm}^{2}$ or $1.9 \%$.

\section{Micro-CT versus $H R C T$ of airways}

A total of 59 Ai measurements from micro-CT and HRCT were compared. The mean Ai from micro-CT was $24.1 \pm 5.7 \mathrm{~mm}^{2}$ (idealised diameter $5.1 \pm 0.5 \mathrm{~mm}$ ) and ranged $1.7-80.9 \mathrm{~mm}^{2}$. There was a systematic and size-dependent underestimation of Ai when measured using HRCT. This was linear in nature (fig. 6a). From a plot of micro-CT Ai and HRCT Ai (data not shown; $\mathrm{r}^{2}=0.96, \mathrm{p}<0.0001$ ), the difference between the Ai measured from both methods was shown to fit the following equation:
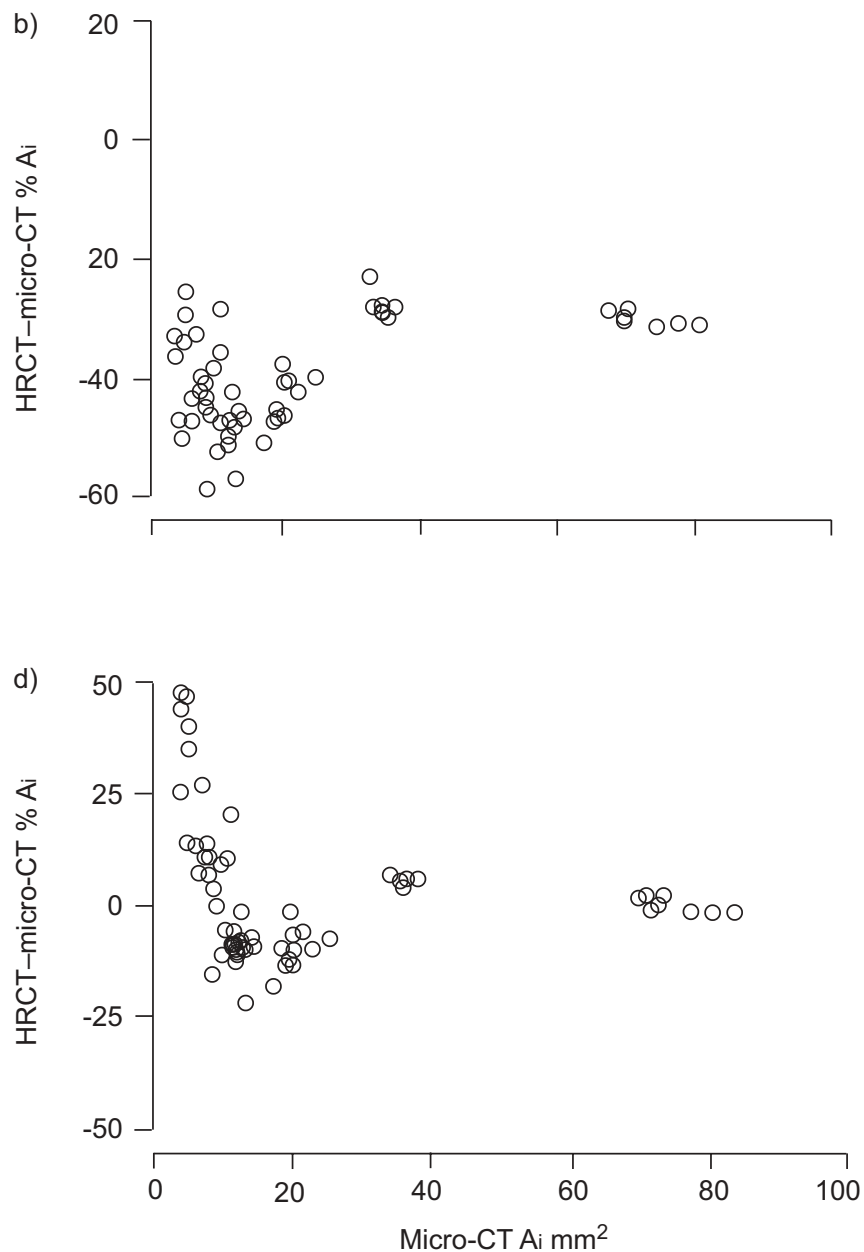

FIGURE 6. Comparison of lumen area (Ai) measurements made by high-resolution computed tomography (HRCT) and micro-computed tomography (CT). a, b) A systematic, size-dependent underestimation of Ai by HRCT was present before calibration of HRCT data; c, d) after calibration, the magnitude of the errors was independent of airway size. ----: mean difference; - - -: 95\% limits of agreement. 

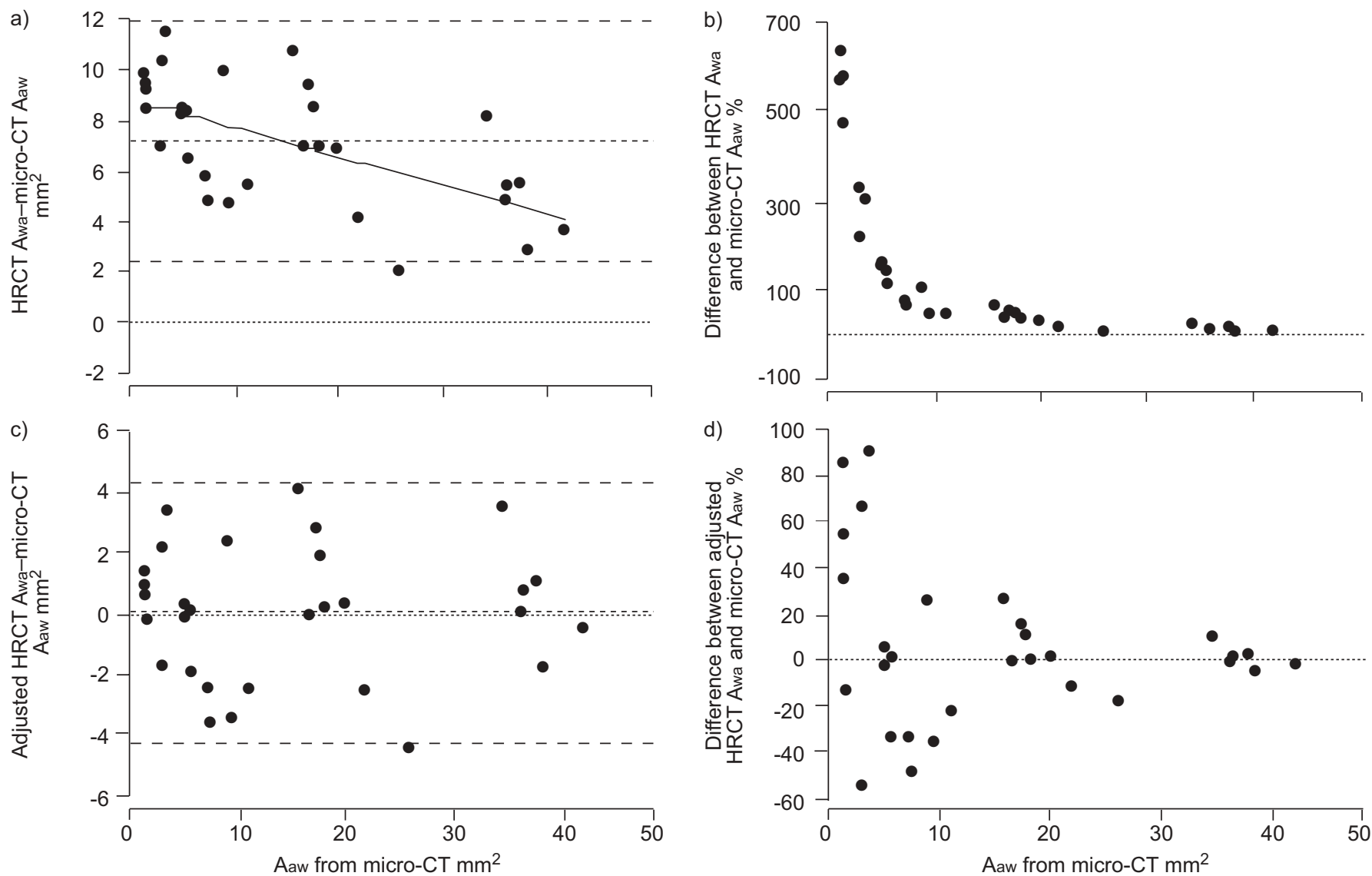

FIGURE 7. Comparison of airway wall area (Aaw) measurements made by high-resolution computed tomography (HRCT) and micro-computed tomography (CT). a, b) A systematic overestimation of Aaw by HRCT was present that was inversely related to true wall area; c) after calibration, the magnitude of the errors was independent of airway size; d) expected the percentage errors were greater for smaller airways. ----: mean difference; - - -: $95 \%$ limits of agreement.

HRCT $\mathrm{Ai}=0.7224 \times$ micro-CT Ai-1.7241

After this equation, or calibration factor, was applied to all HRCT Ai measurements, the $95 \%$ limits of agreement of $\mathrm{A}$ measurement were $\pm 3.2 \mathrm{~mm}^{2}$ (fig. 6c). Percentage errors (fig. $6 \mathrm{~b}$ and $\mathrm{d}$ ) were, as expected, larger for smaller airways. The percentage error was greatest in airways with $\mathrm{Ai}<10 \mathrm{~mm}^{2}$ (internal diameter $<3.5 \mathrm{~mm}$ ).

A total of 30 airway wall area measurements from micro-CT and HRCT were compared (mean Ai $29.4 \pm 9.6 \mathrm{~mm}^{2}$, idealised diameter $6.5 \pm 1.0 \mathrm{~mm})$. Airways with Aaw $<10 \mathrm{~mm}^{2}$ could not
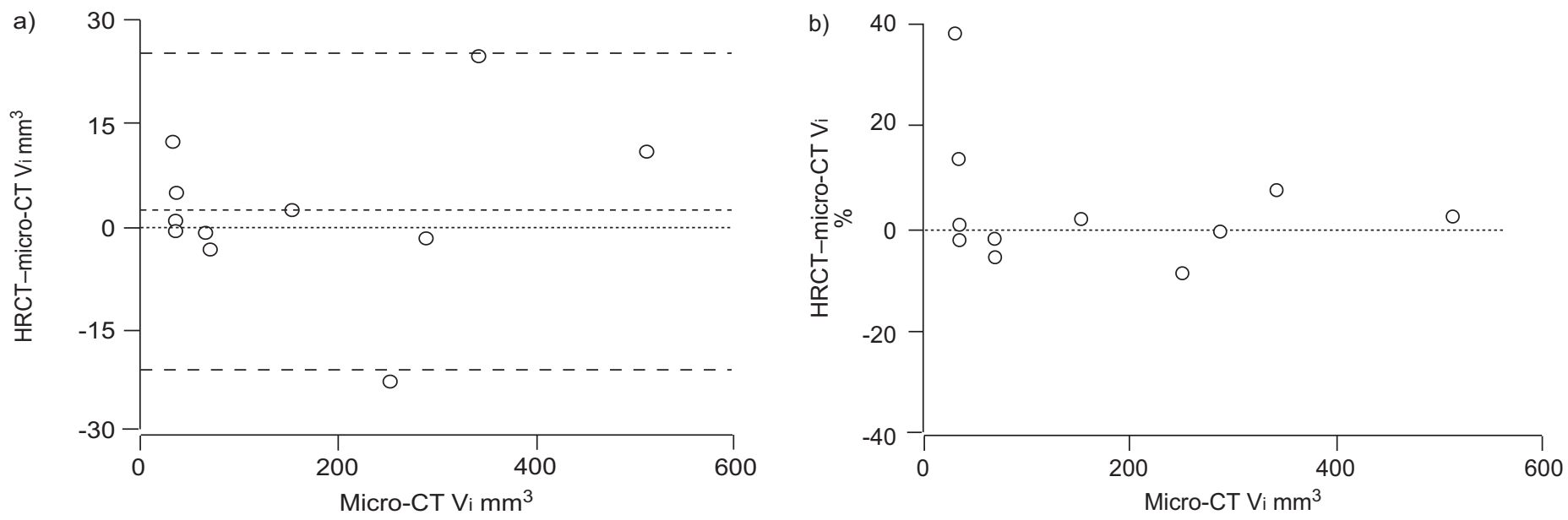

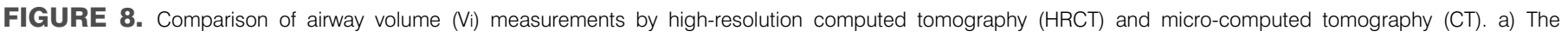
magnitude of error was not size dependent; b) percentage errors were greatest for small airways. ----: mean difference; -- -: 95\% limits of agreement. 
be segmented for wall area reliably with the current authors' segmentation algorithm, and were therefore excluded from the analysis. The excluded airways had a corresponding $\mathrm{Ai}$ $<5 \mathrm{~mm}^{2}$, and an idealised diameter of $<2.5 \mathrm{~mm}$. The overestimation of Aaw when measured using HRCT was size dependent and linear (fig. 7a). From a plot of micro-CT Aaw and HRCT Aaw (data not shown; $\mathrm{r}^{2}=0.97$; $\mathrm{p}<0.0001$ ), the difference in Aaw was found to fit the following equation:

HRCT Aaw $=0.8872 \times$ micro-CT Aaw +8.8611

After calibration of the HRCT Aaw measurements, the 95\% limits of agreement of Aaw measurements were $\pm 4.3 \mathrm{~mm}^{2}$ (fig. 7c). Percentage errors were again larger for smaller airways (fig. $7 \mathrm{~b}$ and $\mathrm{d}$ ). The percentage error was greatest in airways with an Aaw $<10 \mathrm{~mm}^{2}$.

Airway volume was measured using the calibrated segmentation algorithm for all airways that had at least three consecutive HRCT images in which segmentation could be done. Airway lumen volume was calculated as the product of voxel volume $(0.35 \times 0.35 \times 1.25 \mathrm{~mm})$ and the number of voxels within the lumen from all contiguous slices along the airway volume. Volume measurements from HRCT images from 11 airways using the calibrated measurements agreed closely with micro-CT Vi measurements with no systematic error or size dependence (fig. 8a). The $95 \%$ limits of agreement were $\pm 11.2 \mathrm{~mm}^{3}$. Again, when errors were expressed as a percentage of airway volume, the relative errors were larger for smaller airways (fig. 8b).

\section{DISCUSSION}

In this study, 2D and 3D measurements of the airway lumen were obtained from micro-CT images of explanted lung tissue to serve as calibration standards. An inflation fixation was used that kept the tissue at a similar density to what it would have been in vivo, before morphometry, HRCT scanning and micro-CT scanning of the same tissue was undertaken. By using micro-CT, the previous limitation of having only $2 \mathrm{D}$ calibration standard measurements (i.e. lumen area) of explanted airways was overcome. The current authors' $\mathrm{Ai}$ segmentation method for HRCT data resulted in significant underestimation when compared to the calibration standard measurements. The measurement error was predictable, being size dependent in a linear fashion. The current authors' segmentation algorithm for HRCT was also found to result in a linear, size-dependent overestimation of Aaw. The calibration of the computerised segmentation algorithm resulted in $\mathrm{Ai}$ and Aaw measurements from HRCT that were free of systematic errors and allowed determination of the overall precision of the current authors' measurement tool. The close agreement and lack of systematic error between micro-CT and morphometric measurements of Ai suggest that micro-CT measurements do not suffer any significant volume averaging. Validation of the current authors' ray-casting segmentation for HRCT against micro-CT produced accurate Vi measurements with relatively small and unbiased errors.

There are several possible sources of errors in airway measurements from HRCT image data. These include volume averaging (which can be exacerbated by more acute angles of airway orientation relative to the scanning plane); the scanner's point spread functions and reconstruction algorithms; and the lumen segmentation methods [19]. It is possible to measure the angles of orientation of airways from volumetric HRCT data to correct for volume averaging [20], potentially reducing variation in HRCT measurements. The present authors were unable to do this with the current iteration of their analysis algorithm, but it needs to be addressed in future studies. It is likely that these sources of error may differ between manufacturers and models of HRCT machines. Therefore, it may be useful to examine the variations in CT image data arising from differences in clinical CT scanners, as well as those arising from differences in reconstruction algorithms. The magnitude of the variation is not known, nor is it known whether it is clinically significant, although it is common for serial chest CT scans for monitoring disease to be carried out using different scanners.

The manual outlining of airway lumen borders on micro-CT image data might have been an additional source of error, but the present authors think this is likely to be very small. This is because the very small errors in measurements of diameter obtained from the cylindrical rods are an order of magnitude smaller than the errors introduced through HRCT image acquisition and analysis. Measurements of $\mathrm{Ai}, \mathrm{Aaw}$ and $\mathrm{Vi}$ made from micro-CT were unaffected by window settings because of the great spatial resolution and the great contrast at the lumen interface. It is interesting that the $95 \%$ limits of agreement between micro-CT and surface morphometry $\left( \pm 2.5 \mathrm{~mm}^{2}\right)$ were similar to the agreement between micro-CT and HRCT $\left( \pm 3.2 \mathrm{~mm}^{2}\right)$. The disagreement between micro-CT and surface morphometry may be due to the reconstruction plane of micro-CT being slightly out of alignment with the cut surface of the lung which was photographed. The implication is that similar errors are likely to have occurred when comparing micro-CT with HRCT and this also suggests that the comparison method could be improved in the future by using a $3 \mathrm{D}$ registration process to remove this potential source of error. It should also be noted that in some cases, the airways tended to taper towards the surface of the lung cubes, with the airway reaching its largest diameter toward the centre of the lung cube. These airways were not included in the comparison between micro-CT and morphometry, because they were not visible on the cut surface of the lung.

Volume averaging is the factor most likely to account for the bulk of the errors in Ai measurements. The effects of volume averaging on apparent $\mathrm{Ai}$ are likely to be greatest in the z-axis in HRCT, because the z-dimension of the HRCT voxel $(1.25 \mathrm{~mm})$ is almost four times as long as the $\mathrm{x}$ - and $\mathrm{y}$ dimensions $(0.35 \mathrm{~mm})$. This is relevant because airways are usually not parallel to the z-axis in human HRCT studies, nor were they in the current study. As a result, the effects of volume averaging and, hence, the magnitude of the underestimation of lumen area are greater as airway size decreases and the angle of orientation increases (fig. 1) [8, 19, 21]. The use of micro-CT for calibration allowed the current authors to account for the effects of volume averaging, which would not have been possible with 2D measurements. Furthermore, when spiral CT is used, rather than conventional axial HRCT, there is a blurring of structures in the z-axis due to the continuous movement of patients through the rotating tube. Finally, Ai, Aaw and $\mathrm{Vi}$ were measured from HRCT image data in Hounsfield Units, a measure of tissue density [19]. They were, 
therefore, unaffected by window level or width settings, which can affect visual analyses of photographed images [11, 22].

The current authors found that the lower limit of airway size for which lumen measurements could be made with accuracy, in terms of percentage errors, was $\sim 10 \mathrm{~mm}^{2}$. This corresponds to an idealised internal diameter of $3.5 \mathrm{~mm}$ in the current data set, i.e. fourth-generation or segmental airways. Airway wall area measurements were accurate in airways with wall areas $\sim>10 \mathrm{~mm}^{2}$. This corresponds to airways with internal diameters of $\sim 2.5 \mathrm{~mm}$, i.e. fifth-generation or sub-segmental airways. The implication is that greater numbers of airways need to be measured when looking at any percentage changes in Ai or Aaw associated with interventions, for airways smaller than those specified previously. However, there is greater power if absolute changes in either $\mathrm{Ai}_{\mathrm{i}}$ or Aaw are being measured. It is essential to be able to correct or calibrate the measurements made from HRCT, and to know the accuracy of the subsequent calibrated measurements so that studies that are designed to look for changes in airway dimensions are powered appropriately. For instance, the current authors found that even after calibration, smaller airways continued to have large errors associated with them when expressed as a percentage of airway size. Consequently, a much larger number of small airways would need to be studied in patients to detect a real effect of an intervention on smaller airway dimensions than in larger airways where the error as a percentage of total area is much smaller.

The current authors used one airway segmentation method for HRCT, although there are many airway analysis algorithms, all of which are based on slightly different segmentation criteria $[1,3-8,21]$. In the present study, the point at which the density change over two pixels was maximal along the radial spike was used, while others have used the more common "full width at half-max" method. The former method is likely to result in a greater underestimation of the lumen, but it was chosen for the current study because it appeared to segment the lumen reliably even in small airways. Another possible factor is the number of radial spikes used in the segmentation. The ideal number may vary depending on the edge-detection method and is worthy of further study. Such systematic errors relating to a particular methodology of finding the airway lumen edge can be corrected by comparison with calibration standards generated by studies, such as the present one. The precision of different segmentation methods can then be meaningfully compared. The data set of HRCT airway images could be used in the future to compare, and possibly standardise, measurements made using the different segmentation algorithms. Given the increasing likelihood that computerised segmentation will be used routinely in clinical practice to measure airway dimensions, documentation of the differences in $\mathrm{Ai}$ and Aaw measurements associated with different segmentation methods needs to be done using calibration standards in follow-up studies.

The formalin steam fixation method did not significantly alter lung density, which makes this validation method likely to be more accurate and relevant to in vivo lung imaging than validation with artificial material phantoms. Lung densities pre- and post-fixation were $0.34 \mathrm{~g} \cdot \mathrm{mL}^{-1}$ and $0.38 \mathrm{~g} \cdot \mathrm{mL}^{-1}$, respectively. The tissue density of the formalin-fixed pig lung was compared with human in vivo lung as measured by HRCT and found to be similar. The lung cubes were very delicate and needed to be handled extremely gently as they were moist and not completely stiff. A very sharp and thin knife was used to slice the lung, but a slight distortion of the parenchyma at the site of slicing was noticed in the lung cubes. Since all of the analysis was performed after slicing, the effect of the small distortions at the sliced edge was constant across imaging techniques and not considered an important issue. Wet fixation (a method commonly used for excised lungs, in which formalin solution is pumped into the lung or lobe) was considered unsuitable for this validation because it results in fluid-filled alveoli, thereby altering mean lung density, as well as introducing additional fluid into the airway lining. Dry fixation was similarly considered to be unsuitable because it causes desiccation of the lung. Therefore, the calibration standard described in the present study gives a more "real life" measure of the artefacts in airway appearance and measurement that would occur in HRCT scans of human subjects. Another strength of the present study is that the micro-CT, morphometry and HRCT were performed after fixation, which enabled the current authors to determine measurement errors that were associated with image analysis, since there could be no errors due to fixation artefacts.

HRCT provides the unique opportunity to monitor the response of airways to treatment or other stimuli by making direct measurements on airways in vivo over time. It is essential that airway measurements are accurate and that the accuracy of the measurements is independent of airway size, since an airway that is reduced in size owing to moderate or severe increases in smooth muscle tone, inflammation or wall thickening, may experience large changes in lumen size if the inflammation and bronchoconstriction resolve. If the accuracy of the measurements is largely size dependent, then the magnitude of the change in airway dimensions will be masked. It is widely accepted that HRCT measurements of lumen area tend to underestimate actual lumen area in a size-dependent fashion such that the absolute error increases as airway size increases, and HRCT measurements of airway wall tend to overestimate actual airway wall dimensions in the same sizedependent fashion [8]. Therefore, if a patient was scanned during an acute exacerbation of asthma while the airways were inflamed and constricted, then scanned again after recovery and treatment with anti-inflammatory medication, the errors associated with the two scans of the same airway would differ greatly. This process also works in reverse, such that the underestimation of airway lumen size before bronchoconstriction will be larger than the underestimation error associated with the same airway measured after constriction, so the absolute change in lumen area would itself be underestimated.

In the present study, a software standard, not a hardware standard, has been developed. A data set of micro-CT and HRCT images has been created. This can be provided to other researchers to be analysed using their own segmentation software. This is not a hardware comparison tool for CT scanners. In order to make this method applicable to other scanners, a better, longer-lasting method for preserving the lung tissue samples after they are fixed needs to be developed so that the lung cubes can be sent to different research sites to be scanned by different scanners. The paraffin wax used to seal 
the cubes in the present study is air permeable, and the current authors found that after a number of weeks the tissue had shrunk noticeably when the cubes were scanned again.

In summary, the current authors have used micro-computed tomography as the standard to calibrate and then measure the accuracy of a computerised segmentation algorithm for measuring lumen area, wall area and lumen volume using high-resolution computed tomography. The importance of this work is in providing a three-dimensional standard for calibration, which allows more accurate in vivo measurements to be made of airway dimensions from using high-resolution computed tomography. This will allow clinicians and researchers to assess airway pathophysiology more effectively. The micro-computed tomography method is more technically complex and only available in large academic centres, but clearly its value is as a three-dimensional calibration standard which allows comparison of different models of CT scanner, reconstruction methods and segmentation algorithms. The current authors' segmentation algorithm, which utilised a radial-spike method, was shown to underestimate lumen area and overestimate wall area in a size-dependent manner, which is in keeping with results of previous studies by the current authors and others. Having an unbiased three-dimensional method will allow researchers to obtain important data on airway lengths, lumen areas and wall areas that are useful, for instance, in modelling studies and in longitudinal treatment studies. The close agreement between morphometry and micro-computed tomography measurements suggests that the two methods are comparable for use as two-dimensional standards. The ultimate goal is that a standard calibration method can be used between research groups to address differences in airway measurements due to different hardware, scanning methodology and reconstruction, and segmentation algorithms.

\section{REFERENCES}

1 Venkatraman R, Raman R, Raman B, et al. Fully automated system for three-dimensional bronchial morphology analysis using volumetric multidetector computed tomography of the chest. J Digit Imaging 2006; 19: 132-139.

2 Brown RH, Herold CJ, Hirshman CA, Zerhouni EA, Mitzner W. In vivo measurements of airway reactivity using high-resolution computed tomography. Am Rev Respir Dis 1991; 144: 208-212.

3 Wood SA, Zerhouni EA, Hoford JD, Hoffman EA, Mitzner W. Measurement of three-dimensional lung tree structures by using computed tomography. J Appl Physiol 1995; 79: 1687-1697.

4 Amirav I, Kramer SS, Grunstein MM, Hoffman EA. Assessment of methacholine-induced airway constriction by ultrafast high-resolution computed tomography. J Appl Physiol 1993; 75: 2239-2250.

5 Herold CJ, Brown RH, Mitzner W, Links JM, Hirshman CA, Zerhouni EA. Assessment of pulmonary airway reactivity with high-resolution CT. Radiology 1991; 181: 369-374.

6 Okazawa M, Muller N, McNamara AE, Child S, Verburgt L, Pare PD. Human airway narrowing measured using high resolution computed tomography. Am J Respir Crit Care Med 1996; 154: 1557-1562.
7 Niimi A, Matsumoto H, Amitani R, et al. Airway wall thickness in asthma assessed by computed tomography. Relation to clinical indices. Am J Respir Crit Care Med 2000; 162: 1518-1523.

8 King GG, Muller NL, Whittall KP, Xiang QS, Pare PD. An analysis algorithm for measuring airway lumen and wall areas from high-resolution computed tomographic data. Am J Respir Crit Care Med 2000; 161: 574-580.

9 Goris ML, Zhu HJ, Blankenberg FG, Chan F, Robinson TE. An automated approach to quantitative air trapping measurements in mild CF lung disease. Chest 2003; 123: 1655-1663.

10 McNitt-Gray MF, Goldin JG, Johnson TD, Tashkin DP, Aberle DR. Development and testing of image-processing methods for the quantitative assessment of airway hyperresponsiveness from high-resolution CT images. J Comput Assist Tomogr 1997; 21: 939-947.

11 McNamara AE, Muller NL, Okazawa M, Arntorp J, Wiggs BR, Pare PD. Airway narrowing in excised canine lungs measured by high-resolution computed tomography. J Appl Physiol 1992; 73: 307-316.

12 Reinhardt JM, D'Souza ND, Hoffman EA. Accurate measurement of intrathoracic airways. IEEE Trans Med Imaging 1997; 16: 820-827.

13 Nakano Y, Whittall KP, Kalloger SE, et al. Development and validation of human airway analysis algorithm using multidetector row CT. In: Clough AV, Chen C-T, eds. Medical Imaging 2002: Physiology and Function from Multidimensional Images. Proceedings of SPIE, the International Society for Optical Engineering. Vol. 4683. Bellingham, SPIE, 2002; pp. 460-469.

14 Gevenois PA, Koob MC, Jacobovitz D, De Vuyst P, Yernault JC, Struyven J. Whole lung sections for computed tomographic-pathologic correlations. Modified GoughWentworth technique. Invest Radiol 1993; 28: 242-246.

15 Wiebel ER, Vidone RA. Fixation of the lung by formalin steam in a controlled state of air inflation. Am Rev Respir Dis 1961; 84: 856-861.

16 King GG, Carroll JD, Muller NL, et al. Heterogeneity of narrowing in normal and asthmatic airways measured by HRCT. Eur Respir J 2004; 24: 211-218.

17 Bland JM, Altman DG. Measurement error. BMJ 1996; 313: 744.

18 Bland JM, Altman DG. Statistical methods for assessing agreement between two methods of clinical measurement. Lancet 1986; 1: 307-310.

19 King GG, Muller NL, Pare PD. Evaluation of airways in obstructive pulmonary disease using high-resolution computed tomography. Am J Respir Crit Care Med 1999; 159: 992-1004.

20 Saba OI, Hoffman EA, Reinhardt JM. Maximizing quantitative accuracy of lung airway lumen and wall measures obtained from X-ray CT imaging. J Appl Physiol 2003; 95: 1063-1075.

21 Brown RH, Croisille P, Mudge B, Diemer FB, Permutt S, Togias A. Airway narrowing in healthy humans inhaling methacholine without deep inspirations demonstrated by HRCT. Am J Respir Crit Care Med 2000; 161: 1256-1263.

22 Bankier AA, Fleischmann D, Mallek R, et al. Bronchial wall thickness: appropriate window settings for thin-section CT and radiologic-anatomic correlation. Radiology 1996; 199: 831-836. 